\title{
SELF-MODULATED HALFTONES
}

\author{
Basak Oztan and Gaurav Sharma \\ ECE Dept., University of Rochester, Rochester, NY, 14627-0126 \\ $\{$ basak, gsharma\}@ece.rochester.edu
}

\begin{abstract}
We propose an analytic method to overcome the trade-off between the spatial and tonal resolution of traditional clustered dot halftones. Continuous phase modulated halftones that allow variations in screen frequency in different regions of the printed image are employed and halftone screen frequency is varied according to the frequency content of the image to be halftoned. The method, which we term self-modulated halftoning, has a computational complexity similar to screening and is significantly lower than that of other adaptive methods that have previously been used to address the same problem. We demonstrate the experimental performance of selfmodulated halftones and discuss its capabilities and limita-
\end{abstract} tions.

Index Terms - Digital halftoning, frequency modulation, gray tone vs. spatial resolution trade-off

\section{INTRODUCTION}

Most hardcopy printing systems commonly employ halftoning to represent a continuous tone (contone) image by a bilevel image such that the bi-level image gives the same perception as the contone image when viewed from a suitable distance. Xerographic and lithographic printing systems, which are the primary choices for high volume printers, use clustered dot halftones since these are stable and reproducible on these printing systems [1]. Clustered dot halftones are known as amplitude modulated $(A M)$ halftones in which the screen is composed of periodic cells and the amount of ink deposited within a cell varies according to the gray level of the image.

Clustered dot halftone screens are primarily characterized by the number halftone cells that can be fit within a linear inch, which is called the screen frequency $f$ and it is typically measured in cells per inch cpi. Traditionally, the resolution of the printer is fixed, but the screen frequency can be changed according to the image to be halftoned [2]. The process involves a trade-off between the spatial resolution and the tonal resolution, i.e. the number of different gray levels that can be reproduced [1, pp. 399-400]. Low frequency screens can offer more tonal resolution than the high resolution screens; on the contrary, high frequency screens allow better reproduc-

This work is partly supported by a gift from the Xerox Foundation and by a grant from the New York State Office of Science, Technology \& Academic Research (NYSTAR). tion of image details. Typically, the screen frequency is chosen such that it is low enough to reproduce sufficient number of gray levels to match the gray level of the contone image without making the clusters visible to human eye from a typical observation distance and high enough to exhibit enough spatial detail of the image without introducing any visible artifacts such as contouring or gray-tone mismatch. To overcome this trade-off, low screen frequencies can be employed at smoother regions of the image such as background and high screen frequencies can be used at spatially varying regions of the image such as edges.

Conventional clustered dot halftones were generated optically and therefore constrained to a constant screen frequency by technology limitations. With the memory and computation power available in current systems, varying the screen frequency within the halftone image becomes more feasible. In this paper, we propose an analytical method to modify halftone screen frequency along the halftone image according to the frequency content of the contone image. The rest of the paper is organized as follows: in Sec. 2 we summarize the existing work on this problem, then in Sec. 3 we present how self-modulated halftones are generated. We show results in Sec. 4 and finally in Sec. 5 we present conclusions and a discussion.

\section{RELATED WORK}

Varying the halftone cell size within the halftone image has been proposed previously for different applications. Three adaptive halftoning schemes to overcome the trade-off between spatial and tonal resolution were proposed by Hel-Or et al. in [3]. All schemes vary the halftone cell sizes adaptively based on a busyness measure of the image, which is computed by filtering the image with a Laplacian kernel. In the first algorithm, 3 different size halftone threshold arrays are designed and depending on the busyness measure of each pixel, one of the threshold arrays are chosen to represent that pixel. Their second solution to the problem is to use continuous cell sizes instead of using certain discrete values. The edges of each rectangular cell are replaced by springs having spring constants determined by the busyness measure of the pixel. Using the spring constants and the positions of cell centers of their corresponding neighbors, the halftone cell is warped into a tetragonal cell iteratively until a stable state is established. Their last solution to the problem was to use Voronoi 
tesselations for the cell centers distributed according to the busyness measure of the image. The halftone cells are not necessarily tetragonal in this scheme due to the Voronoi tesselation. This algorithm gives better results compared to the others, however depending on the size of the Voronoi cells, it may be required to generate isolated dots neighbor to each other which requires a high stability within the printer.

Another application for varying the halftone cell size was proposed by Ostromoukhov et al. [4] for anti-counterfeiting prevention. The spatial domain is warped by a sinusoidal waveform and depending the value of the waveform large or small size halftone cells are produced.

Recently, the use of continuous phase modulation has also been proposed by Oztan et al. [5] for halftone data embedding. An analytic halftone threshold function incorporating phase and frequency modulation is used to generate frequency modulated halftones. To prevent the generation visible artifacts due to phase discontinuities of the clustered continuity of the modulation functions is required, thus the technique is called continuous phase modulated halftones. In the current study, we build on the foundations of this paper and modulate the halftone screen frequency using frequency content of the contone image.

\section{SELF-MODULATED HALFTONES}

Overview of self-modulated halftone generation is shown in Fig. 1. We begin with image content analysis of the contone image and generate two functions $\Psi_{X}(x, y)$ and $\Psi_{Y}(x, y)$ as a function of the frequency content of the image along $x$ and $y$ directions, respectively. These functions are then used to modulate the frequency of the analytical halftone threshold function along $x$ and $y$ directions on the next step and finally the halftone image is generated by thresholding this function with the contone image values. Given this nature of the system, first in Sec. 3.1 we describe our analytic halftone generation and how this system is modified to incorporate frequency modulation and then in Sec. 3.2 we how to generate the modulator functions $\Psi_{\mathbf{X}}(x, y)$ and $\Psi_{\mathbf{Y}}(x, y)$ using image content analysis.

\subsection{Analytic Halftone Generation with Continuous Phase Modulation}

Thresholding the image values $\tau(x, y)$ by a periodic halftone threshold function $K_{T}(x, y)$ is the simplest and the most common technique to generate clustered dot halftone screens. The threshold array can either be pre-designed for a specific halftone screen frequency, or it can be generated dynamically by using an analytic halftone threshold function. Such a function is defined by Pellar $[6,7]$ as:

$$
K_{T}(x, y)=2 \cos \left(2 \pi f_{x} x\right) \times \cos \left(2 \pi f_{y} y\right),
$$

where $f_{x}$ and $f_{y}$ represent the frequencies along the orthogonal $x$ and $y$ axes, representing horizontal and vertical directions, respectively.

Spatially varying phase terms $\Psi_{\mathbf{X}}(x, y)$ and $\Psi_{\mathbf{Y}}(x, y)$ along $x$ and $y$ directions, respectively, are readily incorporated in
Eq. (1) such that the phase of the sinusoids along $x$ and $y$ can be varied. This way, Eq. (1) can be written as [5]:

$$
\begin{aligned}
K_{T}(x, y)= & 2 \cos \left(2 \pi f_{x} x+\Psi_{\mathbf{X}}(x, y)\right) \times \\
& \cos \left(2 \pi f_{y} y+\Psi_{\mathbf{Y}}(x, y)\right) .
\end{aligned}
$$

To avoid any visible artifacts the continuity of the phase terms are required, thus the technique is called continuous phase modulated halftoning [5].

Although it is difficult to obtain the instantaneous frequencies along $x$ and $y$ directions using this formulation, as it is also shown in [5], if the phase terms $\Psi_{\mathbf{X}}(x, y)$ and $\Psi_{\mathbf{Y}}(x, y)$ do not vary along $y$ and $x$ directions, respectively, the threshold function becomes a separable function and the instantaneous frequencies along $x$ and $y$ directions can be computed as:

$$
\begin{aligned}
f_{i x}(x, y) & =f_{x}+\frac{1}{2 \pi} \frac{d \Psi_{\mathbf{X}}(x)}{d x}, \\
f_{i y}(x, y) & =f_{y}+\frac{1}{2 \pi} \frac{d \Psi_{\mathbf{Y}}(y)}{d y} .
\end{aligned}
$$

Based on this formulation, in Sec. 3.2, we compute the phase terms $\Psi_{\mathbf{X}}(x, y)$ and $\Psi_{\mathbf{Y}}(x, y)$ such that the instantaneous frequencies $f_{i x}(x, y)$ and $f_{i y}(x, y)$ are functions of the frequency content of the image.

\subsection{Image Frequency Content Estimation}

As described in Sec. 3.1, by using Eq. (2) halftone screen frequency can be varied analytically by the phase terms $\Psi_{\mathbf{X}}(x, y)$ and $\Psi_{\mathbf{Y}}(x, y)$. Based on the nature of the spatial and tonal resolution trade-off described in Sec. 1, we see that it is desirable that the phase modulation functions $\Psi_{\mathbf{X}}$ and $\Psi_{\mathbf{Y}}$ increase halftone frequency in high frequency image regions and decrease in smooth image regions. Accordingly, we propose estimation of these from the frequency content of the image.

The directional DCT energy terms $E_{X}(x, y)$ and $E_{Y}(x, y)$ are computed as a metric to evaluate the image frequency content along the subscripted directions. 2-D DCT is computed around the $1 \mathrm{~cm}$. printed size neighborhood of each pixel $\left(x_{0}, y_{0}\right)$, and the DCT coefficients are weighted along the subscripted with a Hanning window of the form

$$
w[n]=\frac{1}{2}\left(1+\cos \frac{\pi(n-N)}{N}\right) \text { for } 0 \leq n \leq N-1,
$$

where $N$ is the DCT window size. Weightings for $x$ and $y$ directions are painted with gradient gray levels in Fig. 1 for better illustration.

Weighting of DCT coefficients eliminates the DC component within the window and emphasizes on low and high frequencies in ascending order. Then, we compute the normalized energies of the DCT windows and assign these values at $E_{X}\left(x_{0}, y_{0}\right)$ and $E_{Y}\left(x_{0}, y_{0}\right)$.

\subsection{Continuous Phase Modulation of Halftone Frequency}

To generate the phase modulation functions $\Psi_{\mathbf{X}}$ and $\Psi_{\mathbf{Y}}$ in Eq. (2), first we take the gradient and then the integral of 


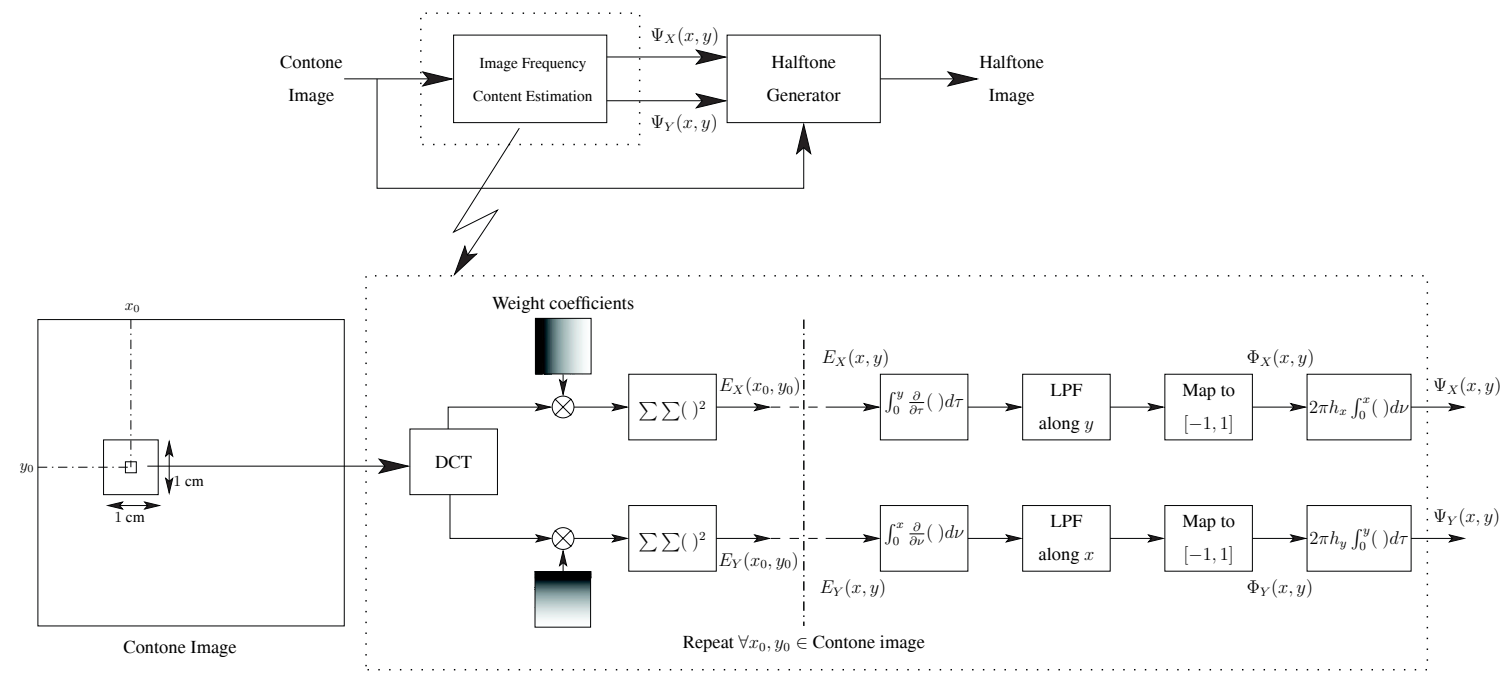

Fig. 1. Overview of self-modulated halftone generation

the directional DCT energy terms along their orthogonal directions (e.g., along $y$ for $E_{\mathbf{X}}$ ). This eliminates the initial values that can lead visible artifacts on the halftone image. In Sec. 3.1, we pointed out separability of these terms are required for the exact computation of the instantaneous frequencies. Since natural images are typically not separable, we obtain approximate local separability by low pass filtering the directional DCT energy terms in the direction orthogonal to their subscripted directions. For this purpose we use Gaussian low pass filters of size $M$. Based on experimental evaluation, we choose $M$ to be $3 \mathrm{~cm}$. printed size of the image in our experiments. In Sec. 5 we discuss the effect of choosing larger and smaller filter on the results.

In the next step, we map the low pass filtered directional DCT energy terms between $[-1,1]$ and finally, the phase terms $\Psi_{\mathbf{X}}(x, y)$ and $\Psi_{\mathbf{Y}}(x, y)$ are found as:

$$
\begin{aligned}
& \Psi_{\mathbf{X}}(x, y)=2 \pi h_{x} \int_{0}^{x} \Phi_{X}(\nu, y) d \nu \\
& \Psi_{\mathbf{Y}}(x, y)=2 \pi h_{y} \int_{0}^{y} \Phi_{Y}(x, \tau) d \tau,
\end{aligned}
$$

where $h_{x}$ and $h_{y}$ are the modulation indices and $\Phi_{X}(x, y)$ and $\Phi_{Y}(x, y)$ are the frequency deviation indices. With this notation, the frequency deviation on the halftone screen along $x$ and $y$ directions can be between $\left[f_{x}-h_{x}, f_{x}+h_{x}\right]$ and $\left[f_{y}-h_{y}, f_{y}+h_{y}\right]$, respectively.

\section{EXPERIMENTAL RESULTS}

To test our algorithm, we use Library image shown in Fig. 2.

We compute the directional DCT energy terms using the method described in Sec. 3. Fig. 3 shows the vertical directional energy term $E_{Y}(x, y)$. The self-modulated halftone image produced using the proposed method is shown in Fig. 4. For generating this image we used $f_{x}=f_{y}=37.5 \mathrm{cpi}$ and

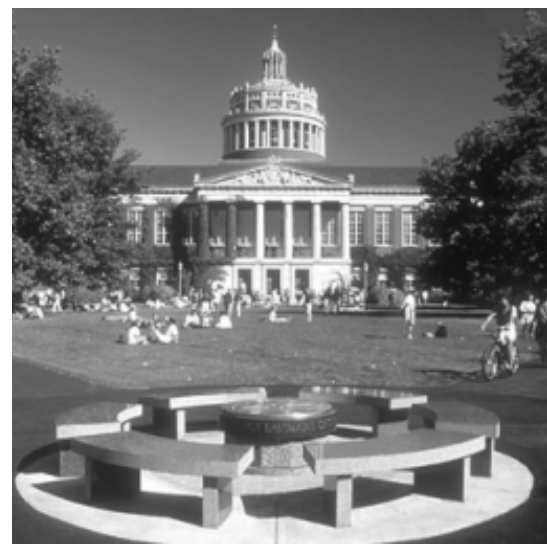

Fig. 2. Contone Library image

$h_{x}=h_{y}=10$ for a $1200 \times 1200$ dpi printer having printed image size $17.34 \mathrm{~cm} . \times 17.34 \mathrm{~cm}$. Due to downsampling of the image to fit in this document, the printed version of this document may not be appropriate for evaluation, however, the actual effect can be seen on the computer screen (by zooming in). In Fig. 5 and Fig. 6 we show enlarged segments from the roof of the building and sky to illustrate the effect of frequency modulation around a high frequency region and a low frequency region, respectively. From the figures it can be seen that the self modulation indeed causes a significant frequency variation in the halftone. Full resolution prints also illustrate that the method alleviates the tonal vs spatial resolution tradeoff.

\section{CONCLUSION}

By using the image to be halftoned to generate the phase modulation signal for continuous phase modulated halftones, we demonstrated a self-modulated halftoning methodology that overcomes the traditional trade-off between spatial and tonal 


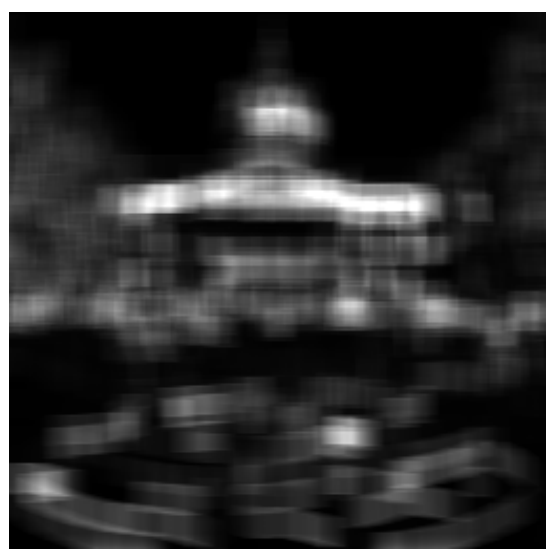

Fig. 3. $E_{Y}(x, y)$ for Library image

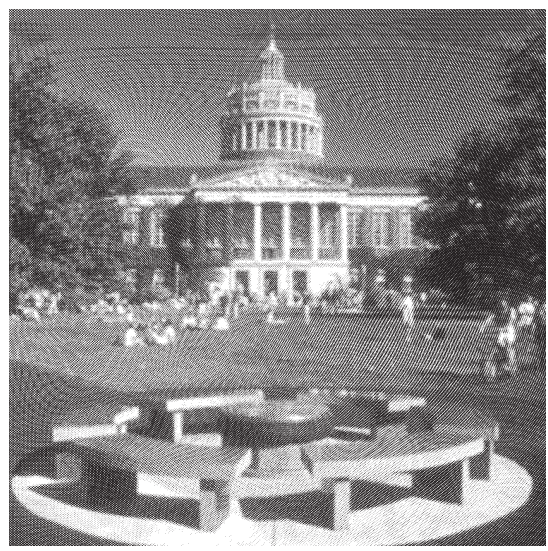

Fig. 4. Self-modulated Library halftone image

resolution

The requirement of separability of the modulation functions poses significant challenges for the method an is subject of ongoing investigation. For example, larger $M$ can impose better separability, however, in that case, the frequency along a single direction is an average of all the frequencies opposite to that direction and it is not desirable for the frequency content of different regions effect each other significantly.

\section{ACKNOWLEDGMENT}

We would like to thank Dr. Robert P. Loce of Xerox Laboratories, Webster, NY for discussions, suggestions, and help with printing high resolution images.

\section{REFERENCES}

[1] C. M. Hains, S. Wang, and K. T. Knox, "Digital color halftones," in Digital Color Imaging Handbook, G. Sharma, Ed. Boca Raton, FL: CRC Press, 2003, chapter 6 .

[2] O. Bryngdahl, "Halftone images: Spatial resolution and tone reproduction," J. Opt. Soc. Am., vol. 68, no. 3, pp. 416-422, Mar. 1977.

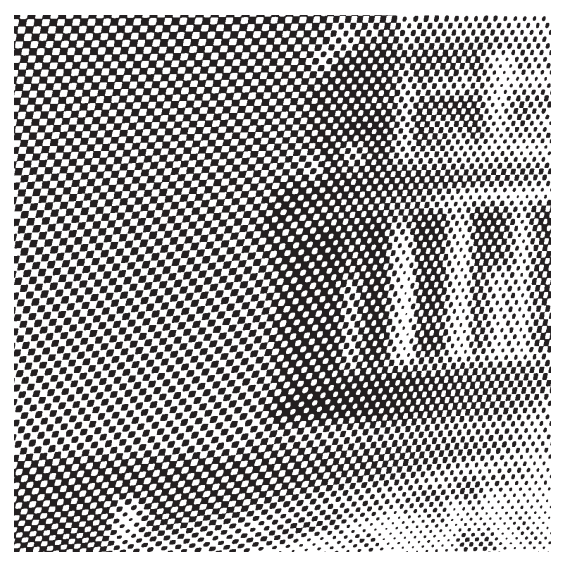

Fig. 5. Enlarged view of a region from Fig. 4. Note the high frequencies in lower right corner.

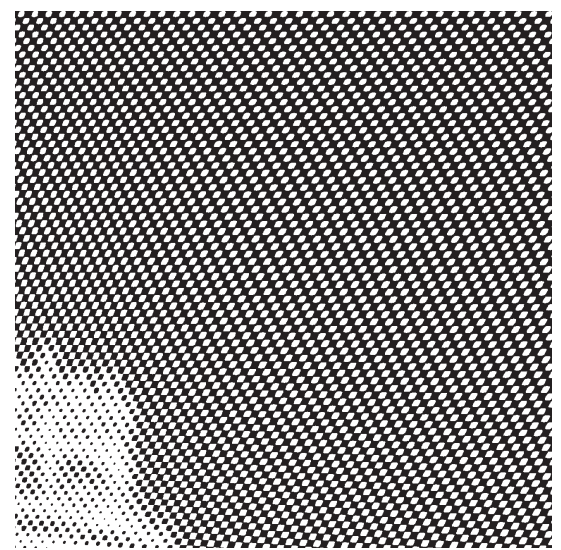

Fig. 6. Enlarged view of a region from Fig. 4. Note the low frequencies in the shadow regions

[3] H. Z. Hel-Or, X. M. Zhang, and B. A. Wandell, "Adaptive clustered dithering," J. Electronic Imaging, vol. 8, no. 2, pp. 133-144, Apr. 1999.

[4] V. Ostromoukhov, N. Rudaz, I. Amidror, P. Emmel, and R. D. Hersch, "Anti-counterfeiting feature of artistic screening," in Proc. SPIE: Holographic and Diffractive Techniques, G. J. Dausmann, Ed., vol. 2951, Oct. 1996, pp. 126-133.

[5] B. Oztan and G. Sharma, "Continuous phase modulated halftones and their application to halftone data embedding," in Proc. IEEE Intl. Conf. Acoustics Speech and Sig. Proc., vol. II, May 2006, pp. 333-336.

[6] R. J. Pellar and L. Green, "Electronic halftone generator," United States Patent No. 4149 183, 1979.

[7] R. J. Pellar, "Electronic halftone generator," United States Patent No. 4196 451, 1980. 